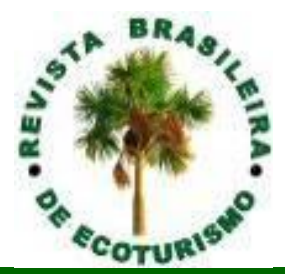

\title{
Validação empírica de um instrumento para mensurar os fatores que afetam o apoio dos residentes ao desenvolvimento do turismo em áreas naturais protegidas
}

\section{Empirical validation of an instrument to measure the factors affecting the residents' support to the development of tourism in protected natural areas}

\section{Carla Stefânia Cabral de Medeiros Santana, Marcos Antônio Leite do Nascimento, Sérgio Marques Junior.}

\begin{abstract}
RESUMO: O objetivo deste artigo é realizar a validação estatística de um instrumento de pesquisa para medir o conjunto de variáveis observáveis pertencentes a cada um dos fatores que podem afetar o apoio dos residentes ao desenvolvimento do turismo na Unidade de Conservação da natureza Parque das Dunas, Natal/ RN. Para tanto, foi desenvolvido um estudo exploratório e descritivo, com abordagem analítica quantitativa. A amostra coletada foi composta de 180 respondentes residentes na localidade de Natal $(\mathrm{RN})$ que se encontravam em visita a área natural. Esta amostra foi coletada de maneira não probabilística por conveniência e utilizando-se a sistematização como critério de seleção dos respondentes. Os dados coletados foram analisados por meio da técnica de estatística multivariada, especificamente análise fatorial exploratória e análise fatorial confirmatória, através do SPSS, v. 18.0. Dos resultados encontrados, conforme os critérios recomendados, conclui-se que estão adequados, consistentes e validados em âmbito exploratório e confirmatório. Desta forma, como contribuição, esta pesquisa possibilitou reforçar a relevância de estudos desta natureza, visando apurar variáveis observáveis na formação de fatores e se estão medindo o que devem efetivamente medir.
\end{abstract}

PALAVRAS CHAVE: Validação; Apoio dos Residentes; Turismo; Unidade de Conservação da Natureza.

ABSTRACT: The objective of this article is to perform the statistical validation of a research instrument to measure the set of observable variables belonging to each factors that may affect the support of residents to the development of tourism in the Nature Conservation Unit Parque das Dunas, Natal/RN. To this end, an exploratory and descriptive study was developed, with a quantitative analytical approach. The sample collected was composed of 180 respondents living in the locality of Natal (RN, Brazil) who were visiting the natural area. This sample was collected in a non-probabilistic manner for convenience and using systematization as a criterion for selecting the respondentes. The collected data were analyzed using the multivariate statistical technique, specifically exploratory factor analysis and confirmatory factor analysis, through SPSS v. 18.0. From the results found, according to the recommended criteria, it is concluded that they are adequate, consistent, and validated in exploratory and confirmatory scope. Thus, as a contribution, this research made it possible to reinforce the relevance of studies of this nature, aiming to determine observable variables in the formation of factors and whether they are measuring what they should effectively measure.

KEYWORDS: Validation; Residents' Support; Tourism; Protected Area. 


\section{Introdução}

Área protegida é uma expressão surgida no final do século XIX devido à intensificação da destruição da natureza e do risco de extinção de espécies, isto, em virtude do acelerado crescimento da sociedade, gerando maior consumo dos recursos naturais. Desta forma, a criação de unidades de conservação está progressivamente se tornando recorrente no cenário mundial, podendo ser definidas como "unidades delimitadas de território, demarcadas para uso humano limitado em nome da proteção de processos naturais e ecológicos" (ZINDA, 2017, p.142).

Inúmeras são as áreas destinadas à proteção dos recursos naturais distribuídas pelo mundo, apresentando as mesmas finalidades, entre elas, minimizar a perda de biodiversidade e melhorar a qualidade de vida da sociedade, visando o reequilíbrio da relação homem-natureza e o desenvolvimento econômico e do turismo (MATHEUS; RAIMUNDO, 2017). Isto porque as áreas naturais protegidas além de conservar o ambiente natural, funcionam como espaços sociais nos quais os incentivos ao turismo aumentam a renda, o emprego e o apoio financeiro para a conservação (OVIEDO-GARCÍA et al., 2019).

No que tange o uso público dessas áreas, principalmente da categoria parque, é crescente a atenção com as formas de intersecção entre o turismo e a conservação ambiental (BUSHELL et al., 2007), sendo esta, em grande parte um fator de atratividade. Neste sentido, é progressivo o número de turistas que se sentem atraídos pelas áreas protegidas devido à importância da proteção ambiental inerente a essas áreas, como destacam Blanco-Cerradelo et al. (2018).

Segundo o World Economic Forum (2017) o Brasil possui a primeira colocação no ranking mundial de recursos naturais, enquanto a Embratur coloca os parques nacionais como reforço do potencial global do Brasil como destino turístico (BRASIL, 2018). O país recebeu no período entre 2016-2017 um total de 6.588.770 turistas internacionais, destes, 16,3\% tinham como motivação a natureza, a aventura e o ecoturismo (BRASIL, 2018), motivação essa que se enquadra com a concepção dos Parques naturais. Enquanto os embarques e desembarques nacionais no ano de 2017 teve o total é de 92.149.646 (BRASIL, 2018) tendo como motivações principais o lazer e a natureza.

Corroborando com esta informação, o Ministério do Meio Ambiente (MMA) (BRASIL, 2016), relatou que houve um crescimento de $238 \%$ nas visitações aos parques nacionais, passando de 2,9 milhões em 2007 para 7,1 milhões em 2015. De maneira global, Ballantyne, Packer e Hughes (2008) contabilizam mais de 250 milhões de visitantes por ano em áreas naturais protegidas.

O potencial turístico dos parques pode estar alusivo às características abióticas e bióticas, culturais e/ou histórica; o enaltecimento de culturas locais; o interesse pessoal por áreas protegidas; a presença de valores singulares; a valorização do meio ambiente; e/ou o surgimento de um novo perfil de turistas. Além de que, normalmente é a forma mais popular que se tem conhecimento quando se pensa em UC (VALLEJO, 2013).

No que tange o turismo e a recreação em UC, Bushell et al. (2007, p. 6) falam que "o turismo e a recreação oferecem benefícios consideráveis para as áreas protegidas e suas comunidades", sendo eles econômicos e sociais, além de uma maior valorização do patrimônio cultural, natural e histórico (MOGHAVVEMI et al., 2017; BUSHELL et al., 2007). 
Contudo, quando mal gerida, a atividade turística contribui para a deterioração da biodiversidade, dos elementos culturais e dos recursos do ecossistema (MOGHAVVEMI et al, 2017; BOWERS, 2016) e consequentemente impacta negativamente na comunidade local (BUSHELL et al., 2007). Podendo também ser geradora de conflitos sociais advindos da exclusão da comunidade, o aumento de episódios de violência no entorno (MOGHAVVEMI et al, 2017; ANDERECK et al., 2005), acumulação de pessoas (GARCIA; VÁZQUEZ; MACíAS, 2015), aumento do custo de vida (MOGHAVVEMl et al., 2017; LEE; BACK, 2006; BESTARD; NADAL, 2007) e distribuição desigual de renda (ALAM; PARAMATI, 2016).

Apesar disso, a verificação do potencial de ganhos para área local e de entorno de uma área protegida advindo do turismo tornou esta atividade "um importante ator nos debates sobre a interação dos discursos neoliberalistas e dos recursos naturais na gestão das áreas protegidas" (DARCY, 1995 apud SCHWEINSBERG; DARCY; CHENG, 2017, P.241).

Sendo assim, reconhecendo o papel do turismo em UC e na comunidade de entorno, bem como, tendo consciência dos impactos positivos e negativos da atividade nestas áreas de conservação, e entendendo que as UCs são áreas que necessitam de um planejamento integrado e participativo (EAGLES; MCCOLL, 2002), compreende-se como relevante a investigação com os residentes locais sobre os fatores que afetam o apoio ao desenvolvimento do turismo em UC. Visto que, para que o turismo prospere em uma área ele precisa do apoio dos residentes (GURSOY et al., 2019; AP; CROMPTON, 1998), isto porque, à medida que os desejos dos moradores são atendidos e suas opiniões são levadas em conta, a receptividade para 0 desenvolvimento do turismo desponta (NUNKOO; RAMKISSOON, 2012).

Ademais, Murphy destaca (1981, p.189), "torna-se necessário considerar as atitudes dos residentes locais para o desenvolvimento do turismo e integrar o público nas decisões de desenvolvimento", uma vez que a comunidade é um dos componentes principais para o turismo nessas áreas (STRICKLAND-MUNRO et al., 2010) e as "que experimentam maior impacto decorrente de áreas naturais protegidas" (STRICKLAND-MUNRO et al., 2010, p. 501).

Assim sendo, o propósito desta pesquisa visa através de método quantitativo validar um instrumento de mensuração que identifique o conjunto de variáveis observáveis pertencentes a cada um dos fatores que podem afetar o apoio dos residentes ao desenvolvimento do turismo em uma Unidade de Conservação de Natal/RN (Brasil). Visando com isso, contribuir para a compreensão da percepção dos residentes perante o apoio ao desenvolvimento da atividade turística nessa área de proteção ambiental, estudo que poucas áreas protegidas dispõem conforme afirma Lilian Hangae, diretora de criação e manejo do ICMBIO (BRASIL, 2016).

Além disso, tem-se também a intenção de que esta validação subsidie uma análise posterior em nível de Modelagem Estrutural a respeito da estimação das múltiplas relações que afetam o apoio dos residentes ao desenvolvimento do turismo na UC Parque das Dunas. 


\section{Unidades de conservação: uma apresentação histórico-conceitual}

A criação do conceito de áreas protegidas se deu inicialmente com o objetivo de proteger "a flora, a fauna e as áreas de interesse natural excepcional" (MCCORNICK, 1992, p. 25) de um determinado território. Na atualidade, outros elementos foram adicionados a esta formação e cada vez mais, cresce no mundo o número de áreas com essas caraterísticas voltadas à proteção, com a responsabilidade da preservação e conservação ambiental.

Com esse crescimento, houve também a necessidade de um aperfeiçoamento e adição de novos elementos aos objetivos iniciais, desta forma, passa-se a ter a preocupação com a diversidade biológica; recursos ambientais como os estuários, o solo, os mares, o subsolo, os elementos da biosfera; habitats; manutenção de ecossistemas; o manejo; o extrativismo e os corredores ecológicos (BRASIL, 2000), e mais recentemente com a geodiversidade e o patrimônio geológico (GRAY, 2004; BRILHA, 2005; NASCIMENTO; RUCHKYS; MANTESSONETO, 2008).

Têm-se relatos históricos que registram que as primeiras experiências no sentido de definir áreas bem delimitadas com a intenção de proteger ambientes, atividades, como a caça, e/ou espécies, remete-se a épocas muito antigas, como por exemplo, na Mesopotâmia (VALLEJO, 2002).

No ocidente, essa configuração, versa sobre a ldade Média, quando havia o destino de áreas para a utilização exclusiva da sociedade feudal e para o reduto de algumas espécies por ordem dos reis (ROCHA, 2002). Nenhum desses registros remete-se a utilização dessas áreas de maneira pública voltada ao lazer.

Com o passar do tempo, novas configurações sociais surgiram, novas rupturas paradigmáticas aconteceram e novos paradigmas foram criados. Desta forma, com a revolução industrial e com 0 aparecimento de movimentos preservacionistas, surge uma composição na forma da população fabril da época que anseia por áreas públicas para o lazer, a fim de ocupar seu tempo de não trabalho.

A partir disso, a concepção de proteção de áreas naturais, principalmente na forma de parques, com a intenção de conservar o ambiente e promover o bem-estar da população em contato com a natureza, começa a tomar uma proporção cada vez maior. Durante muito tempo, os parques eram considerados a forma mais clara e clássica para designar áreas de ecossistemas protegidos, ou seja, todas essas áreas de preservação natural eram parques (DOUROJEANNI, 2015).

O símbolo basilar dessa concepção de áreas protegidas fica estabelecido com o surgimento do Parque Nacional de Yellowstone nos Estados Unidos - EUA em 1872, que tinha como perspectiva a noção de "wilderness" (vida natural selvagem) com a característica de áreas não povoadas, mas com fins de recreação para a população urbana (DIEGUES, 1998).

No Brasil, esse processo de estabelecimento e criação de áreas ambientais protegidas teve início em 1937, e assim como outros países, também utilizou como modelo o Parque de Yellowstone nos EUA. Isto se deu quando o país começou a experimentar um crescimento urbano acelerado e uma ampla industrialização, o que fez com que houvesse um maior interesse em delimitar áreas voltadas à conservação ambiental e proteção cênica. 
O registro mais antigo de área protegida no país data de 1808 com a criação por D. Joao VI do Horto Real, o que hoje é o Jardim Botânico do Rio de Janeiro, alguns anos mais tarde com a elaboração do Código Florestal Brasileiro, surgiu o Parque Nacional de Itatiaia, primeiro parque nacional.

O que antes era tratado pelo Código Florestal Brasileiro, passa a ser regido em 2000 pelo Sistema Nacional de Unidades de Conservação (SNUC) lei n 9.985. Nele definem-se dois grupos de Unidades de Conservação, o grupo das unidades de proteção integral e o grupo do uso sustentável.

O grupo das unidades de proteção integral é formado pelas Estações Ecológicas, Reservas Biológicas; Parques Nacionais; Monumento Natural; e Refúgio de Vida Silvestre. Já as unidades de uso sustentável são formadas pela Área de Proteção Ambiental; Área de Relevante Interesse Ecológico; Floresta Nacional; Reserva Extrativista; Reserva de Fauna; Reserva de Desenvolvimento Sustentável; e Reserva Particular do Patrimônio Natural.

A categoria parque foi criada pelo Código Florestal de 1934, que considerou essas áreas de proteção integral, de uso indireto, não podendo haver exploração de recursos naturais nem residências. Tendo seus objetivos e usos permitidos definidos pelo Art. 11 do SNUC (2000). A visitação em áreas de parques necessita seguir regras bem definidas sob a supervisão do órgão gestor e do plano de manejo, tendo como finalidade a conservação e o uso sustentável dos ecossistemas.

\section{Apoio ao desenvolvimento do turismo e seus antecedentes}

O apoio ao desenvolvimento do turismo tem sido objeto de estudo de inúmeros pesquisadores (AP, 1992; LINDBERG; JOHNSON, 1997; GURSOY; JUROWSKI; UYSAL, 2002; GURSOY; RUTHERFORD, 2004; HALEY; SNAITH; MILLER, 2005; NUNKOO; RAMKISSOON, 2011; NUNKOO; GURSOY, 2012; LEE, 2013; STYLIDIS et al., 2014; GARCIA; VÁZQUEZ; MACÍAS, 2015; MOGHAVVEMI et al., 2017; entre outros). Em grande parte destes estudos, verificam-se as atitudes dos autóctones perante a percepção dos impactos positivos e negativos relacionados aos benefícios e prejuízos locais advindos do turismo no campo da economia, do sociocultural e do ambiental.

Sobre isso, a Teoria das Trocas Sociais (SET) quando inserida no âmbito do turismo, versa sobre a verificação dos benefícios e dos custos e também da busca do benefício mútuo entre atores (comunidade e turistas) (AP, 1992; HALEY; SNAITH; MILLER, 2005; MOGHAVVEMI et al., 2017). A premissa básica é a de que "as pessoas que se beneficiam do turismo percebem menos impactos sociais e ambientais do turismo e têm atitudes mais favoráveis ao desenvolvimento da atividade" (PERDUE; LONG; ALLEN, 1987, p.422) ao contrário daquelas que não possuem qualquer vínculo. Por outro lado, Lindberg e Johnson (1997), ao tratar das atitudes de apoio ao desenvolvimento do turismo, expõem que esta ação depende da importância individual dos valores, ou seja, a formação ou mudança de atitude é derivada da concepção de valor.

Correlacionando esta informação com o comportamento de residentes de destinos turísticos, presume-se que os valores percebidos afetam as atitudes e consequentemente o apoio ao desenvolvimento do turismo. Como expõe Ajzen e Fishbein (1977, p. 888), "as ações das pessoas são encontradas sistematicamente 
relacionadas com suas atitudes quando a natureza dos preditores de atitudes e os critérios comportamentais são levados em consideração".

Quando se pensa na questão do apoio ao desenvolvimento turístico em uma determinada localidade, é comum referir-se apenas aos benefícios econômicos que podem estar atrelados (GURSOY; JUROWSKI; UYSAL, 2002). Stylidis et al. (2014) e Garcia et al. (2015) entendem que se a percepção dos impactos positivos for maior do que os negativos, provavelmente os residentes apoiarão o turismo, da mesma forma que o sucesso do turismo depende do apoio ativo da população local (GURSOY; RUTHERFORD, 2004).

Entretanto, no que se refere à verificação do apoio, Nunkoo e Gursoy (2012) propõem um modelo que agrupa a Teoria das Trocas Sociais com a Teoria da Identidade. Segundo os autores, o apoio ao desenvolvimento do turismo (comportamento) não deve focar apenas na verificação dos impactos, mas também na apuração do vínculo dos indivíduos com a estrutura social, ou seja, "a identidade de um indivíduo influencia no seu comportamento" (NUNKOO; GURSOY, 2012, p.247). Assim como, há outros estudos que sugerem a presença de fatores igualmente importantes que também possam afetar o apoio, tais como a proteção ambiental (LIU; VAR, 1986; LIU; SHELDON; VAR, 1987) e o desenvolvimento comunitário (LEPP, 2007).

A literatura que trata dos antecedentes do apoio ao desenvolvimento do turismo destaca algumas dimensões relevantes para a explicação deste fato, tais como o Valor (LINDBERG; JOHNSON, 1997; GURSOY; JUROWSKI; UYSAL, 2002; LEE, 2013; CHEN; CHEN, 2010; NUNKOO; RAMKISSOON, 2011; NUNKOO; RAMKISSOON, 2012; NUNKOO; GURSOY, 2012; GURSOY; RUTHERFORD, 2004), a Imagem (STYLIDIS et al., 2014; STYLIDIS; SHANI; BELHASSEN, 2017; CHEN; TSAI, 2007; RAMKISSOON; NUNKOO, 2011; SCHROEDER, 1996), a Satisfação (CHEN; CHEN, 2010; CHEN; TSAI, 2007; VARGAS-SÁNCHEZ; PORRAS-BUENO; PLAZA-MEJÍA, 2011; JO; LEE; REISINGER, 2014) e a Qualidade (CHEN; CHEN, 2010; CHEN; TSAI, 2007; JO; LEE ; REISINGER, 2014).

O Valor vem sendo objeto de atenção dos pesquisadores em turismo (LINDBERG; JOHNSON, 1997; CHEN; TSAI, 2007; KASHYAP; BOJANIC, 2000; WANG; ZHANG; GU; ZHEN, 2009; MURPHY; PRITCHARD; SMITH, 2000; OH, 1999; OH, 2000; PETRICK, 2004), pelo fato de que junto com satisfação e qualidade são bons antecedentes de futuras intenções comportamentais, isto para a verificação da intenção de retorno ao destino turístico (CHEN; TSAI, 2007) e do apoio.

Segundo Zeithaml (1988), o Valor, medido de forma individual, possui uma diversidade de significados e por isso a dificuldade em conceituá-lo. Para o autor, Valor pode estar associado ao preço, a benefícios, a qualidade e finalmente, o que você recebe pelo o que é dado. Ou seja, "O valor percebido é a avaliação geral do consumidor da utilidade de um produto com base nas percepções sobre o que é recebido e o que é dado" (ZEITHAML, 1988, p. 14). Apesar da diversidade de conceitos, Woodruff (1997) aborda que existe um consenso, que o valor é inerente à utilidade e a percepção do consumidor, bem como colocado por Zeithaml (1988).

Sendo assim, as percepções dos benefícios influenciam o apoio (LEE, 2013; NUNKOO; RAMKISSON, 2011), que nesse caso, está relacionado ao desenvolvimento do turismo. Sobre isso, Garcia et al. (2015) afirmam que com base na grande maioria dos estudos já realizados, o apoio ao desenvolvimento do turismo 
só ocorrerá se houver por parte das pessoas a percepção de atitudes positivas. Logo, neste estudo a relação Valor-Apoio está alusiva à percepção dos benefícios (prerrogativa das $\mathrm{UC}$ ).

No tocante ao constructo Imagem, este frequentemente é tratado como a representação mental concebida a partir de impressões individuais, tal como também de sentimentos (LEE; LEE; LEE 2005; WANG et al., 2009). Beerli e Martin (2004) consideram que a Imagem geral sobre um atrativo ou destino será formada através das percepções cognitivas e afetivas, e desta forma, relevante para avaliação positiva ou negativa destes. Segundo os autores, outros elementos importantes para a concepção da Imagem são os estímulos sofridos e os fatores pessoais (psicológicos e sociais). Estes, significativos para a verificação do papel das áreas protegidas no cotidiano dos residentes.

Desta forma, Imagem é o constructo que pretende medir o conceito que pode estar atrelado ao atrativo. O comportamento dos residentes e a avaliação da experiência local (LEE; LEE; LEE, 2005) podem ser influenciados pela representação, pela imagem mental que eles possuem, assim como a escolha do lugar como rota de convívio e de frequentação.

O constructo Satisfação é tratado como essencial para o sucesso de muitas organizações, empresas, produtos e serviços. Devido a isso são frequentes as pesquisas de opinião feitas por empresas verificando a satisfação de seus clientes, visto que, não obrigatoriamente, mas normalmente é uma importante atribuição para a fidelização e diminuição da incidência de reclamações, tal como afirmam Fornell et al. (1996). Para turistas, o sentimento de satisfação gera a possibilidade de retorno ao destino ou atrativo e recomendação a amigos e familiares (CHEN; TSAl, 2007). Para UC, a probabilidade de um visitante satisfeito retornar aquela área natural e recomendar aos outros é maior do que um no qual sua expectativa não tenha sido satisfeita.

O constructo Qualidade é tratado por Zeithaml (1988, p.3) como "julgamento do consumidor sobre a excelência ou superioridade global de um produto". Devido essa avaliação global, não obrigatoriamente a percepção da qualidade do serviço gera a satisfação. Pode ocorrer que mesmo que um indivíduo esteja satisfeito com o serviço ainda assim julga que a empresa não prestou uma boa qualidade.

Entretanto, Chen e Tsai (2007) e Jo, Lee e Reisinger (2014) julgam que a qualidade é uma preditora das atitudes comportamentais e desta forma do apoio ao desenvolvimento do turismo. Além disto, "experiências de alta qualidade aos visitantes de parques aumentam o interesse na proteção e conservação dos valores das áreas protegidas" (BUSHELL et al., 2007, p.6)

A apuração da percepção dos benefícios sociais e ambientais, tal como do uso e da qualidade, permite investigar o significado, as interpretações, as relações, os comportamentos, a importância daquela área natural e a influência dela sobre as pessoas, contribuindo dessa forma com a gestão da unidade de conservação (SHIRAISHI, 2011).

Baseando-se nestes conceitos, foi proposto neste estudo validar um instrumento de mensuração dos fatores que podem influenciar o apoio ao desenvolvimento do turismo em uma área natural protegida. Os procedimentos metodológicos adotados são apresentados e discutidos a seguir. 


\section{Metodologia}

A presente investigação caracteriza-se como exploratório e descritivo, com abordagem analítica quantitativa. O objeto do estudo foi a UC Parque das Dunas, Estado do Rio Grande do Norte, Brasil, instalada em um total de 1.172,80 hectares, localizado na malha urbana da capital do Estado, Natal, e próxima ao corredor turístico da cidade. Diariamente, esta unidade recebe visitantes a fim de usufruir das atividades disponíveis no local, assim como, da própria natureza existente. O público da pesquisa em sua totalidade é caracterizado e definido como residentes locais que estavam em visita ao parque.

O instrumento utilizado trata-se de um questionário que contém 32 variáveis observáveis divididas nos fatores Valor, Imagem, Satisfação, Qualidade e Apoio. As questões são medidas em escala métrica de 11 pontos, indo de 0 discordo plenamente a 10 concordo plenamente com uma frase apresentada ao respondente.

Cada um desses fatores é composto por variáveis, como pode ser observado no Quadro 1. Este além de apresentar os fatores com suas respectivas variáveis observáveis expõe os autores que embasaram a construção de cada fator.

Quadro 1: Resumo dos constructos e variáveis de análise.

Frame 1: Summary of constructs and analysis variables.

\begin{tabular}{|c|c|c|}
\hline FATORES & DESCRIÇÃO DAS VARIÁVEIS & AUTORES \\
\hline \multirow{7}{*}{ Valor } & VALOR1- Saber que este parque é uma UC me trouxe aqui & \multirow{7}{*}{$\begin{array}{l}\text { Moreira (2016) } \\
\text { Nunkoo; } \\
\text { Ramkissoon } \\
\text { (2011) } \\
\text { Passold; Kinker } \\
\text { (2010) } \\
\text { Eagles; Mccool } \\
\text { (2002) } \\
\text { Chen; Tsai } \\
\text { (2007); } \\
\text { Wang; Zhang, } \\
\text { Gu; Zhen (2009); }\end{array}$} \\
\hline & $\begin{array}{l}\text { VALOR2- Eu observo que ambientalmente este parque é bem } \\
\text { conservado }\end{array}$ & \\
\hline & VALOR3- Para mim este parque possui elementos atrativos & \\
\hline & VALOR4- A experiência recreativa neste parque é prazerosa & \\
\hline & $\begin{array}{l}\text { VALOR5- Eu percebo que as pessoas contribuem para manter este } \\
\text { parque conservado }\end{array}$ & \\
\hline & VALOR6- Este parque tem potencial turístico & \\
\hline & $\begin{array}{l}\text { VALOR7- Eu percebo esta área como mais um atrativo turístico para a } \\
\text { cidade de Natal }\end{array}$ & \\
\hline \multirow{7}{*}{ Apoio } & $\begin{array}{l}\text { APOIO1- Essa área é importante para o desenvolvimento do turismo } \\
\text { em Natal }\end{array}$ & \multirow{7}{*}{$\begin{array}{l}\text { Passold; Kinker } \\
\text { (2010) } \\
\text { Nunkoo; } \\
\text { Ramkissoon } \\
\text { (2011) } \\
\text { Nunkoo; } \\
\text { Ramkissoon } \\
\text { (2012) } \\
\text { Lindberg; } \\
\text { Johnson (1997) } \\
\text { Lee (2013) }\end{array}$} \\
\hline & $\begin{array}{l}\text { APOIO2- Acredito que as pessoas deveriam se envolver em ações de } \\
\text { proteção dessa área }\end{array}$ & \\
\hline & APOIO3-Eu sou a favor do desenvolvimento do turismo neste parque & \\
\hline & $\begin{array}{l}\text { APOIO4- Eu apoio um maior incentivo à captação de visitantes para } \\
\text { este parque }\end{array}$ & \\
\hline & $\begin{array}{l}\text { APOIO5- Para que haja visitação, eu sou a favor da cobrança de } \\
\text { ingressos para a entrada neste parque }\end{array}$ & \\
\hline & $\begin{array}{l}\text { APOIO6- O governo deveria criar mais parques como este para } \\
\text { favorecer o turismo em Natal }\end{array}$ & \\
\hline & $\begin{array}{l}\text { APOIO7- Eu gostaria que os turistas que estão em Natal visitassem } \\
\text { este parque }\end{array}$ & \\
\hline \multirow{6}{*}{ Imagem } & IMAGEM1- Quando eu penso em Natal eu lembro deste parque & \multirow{6}{*}{$\begin{array}{l}\text { Lee et. al. (2005) } \\
\text { Wang; Zhang; } \\
\text { Gu; Zhen (2009) } \\
\text { Beerli; Martin } \\
\text { (2004) } \\
\text { Chen; Tsai (2007) }\end{array}$} \\
\hline & IMAGEM2 -Esse parque me lembra conservação ambiental & \\
\hline & IMAGEM3- Esse parque me traz boas recordações & \\
\hline & IMAGEM4- Este parque me lembra sensações agradáveis & \\
\hline & IMAGEM5- Vejo este parque como um espaço educativo & \\
\hline & IMAGEM6- Este parque é muito emocionante & \\
\hline
\end{tabular}

Continua... 
continuação.

\begin{tabular}{|c|c|c|}
\hline FATORES & DESCRIÇÃO DAS VARIÁVEIS & AUTORES \\
\hline \multirow{6}{*}{ Satisfação } & SAT1- Os serviços desse parque funcionam como deveriam & \multirow{6}{*}{$\begin{array}{l}\text { Passold; Kinker } \\
\text { (2010) } \\
\text { Moreira (2016) } \\
\text { Chen; Tsai (2007) } \\
\text { Chen; Chen } \\
\text { (2010) }\end{array}$} \\
\hline & SAT2- Este parque é acessível (tem boa localização) & \\
\hline & $\begin{array}{l}\text { SAT3- A visita a este parque contribui para uma maior interação com } \\
\text { a natureza }\end{array}$ & \\
\hline & SAT4- O parque apresenta excelente infraestrutura de apoio turístico & \\
\hline & SAT5-O parque apresenta todas as condições para o lazer & \\
\hline & SAT6- O parque possui tudo o que preciso para me divertir & \\
\hline \multirow{6}{*}{ Qualidade } & QUALI1- Limpeza da área & \multirow{6}{*}{$\begin{array}{l}\text { Moreira (2016) } \\
\text { Chen; Tsai (2007) } \\
\text { Lee et. al. (2005) } \\
\text { Wang; Zang; Gu; } \\
\text { Zhen (2009) } \\
\text { Chen; Chen } \\
\text { (2010) }\end{array}$} \\
\hline & QUALI2- Segurança & \\
\hline & QUALID- Acessibilidade & \\
\hline & QUALI4- Instalações & \\
\hline & QUALI5-Atividades recreativas & \\
\hline & $\begin{array}{l}\text { QUALI6- De maneira geral, como você avalia a qualidade deste } \\
\text { parque? }\end{array}$ & \\
\hline
\end{tabular}

Fonte: Elaboração própria, 2019.

Source: Authors, 2019.

A aplicação do instrumento de pesquisa foi feita no período de 02 a 26 de Novembro de 2017 durante a semana e nos finais de semana. Para a escolha do sujeito foi utilizado o método amostral não probabilístico por conveniência, utilizando-se um procedimento sistemático de coleta: a cada três pessoas abordavase uma. Na totalidade, a amostra coletada foi composta por 180 questionários, sobre isso, Hair et al. (2005) recomendam que no mínimo seja utilizado um número cinco vezes maior que o total de itens a serem avaliados no instrumento. Desta forma, por possuir 32 itens com uma amostra de 180, o estudo atende ao recomendado.

Os dados obtidos tiveram seu processamento realizado através do Statistical Package for the Social Sciences (SPSS, v. 18.0) para a Análise Fatorial Exploratória (AFE) e Análise Fatorial Confirmatória (AFC), que segundo Hair et al. (2005, p.92) trata-se de uma "técnica de interdependência na qual todas as variáveis são simultaneamente consideradas, cada uma relacionada com todas as outras", tendo como principais objetivos resumir e reduzir dados do modelo de mensuração.

\section{Resultados e discussões}

\section{Análise fatorial exploratória e de confiabilidade das dimensões do estudo}

$\mathrm{Na}$ validação do modelo de mensuração, parte-se inicialmente da análise fatorial exploratória (AFE) como técnica de redução das variáveis observáveis e na especificação das que melhor explicam cada constructo, isto conforme atributos recomendados por Hair et al. (2005), Corrar, Paulo e Dias (2009) e Mâroco (2010). Verificam-se as cargas fatoriais das variáveis, o teste Kaiser-Meyer-Olkin - KMO, a significância do teste de esfericidade de Bartlett's, a variância total explicada para cada dimensão, o Eigenvalue, a Comunalidade, a Matriz de Anti-Imagem (MSA) e o Alpha de Cronbach.

A carga fatorial é a "correlação entre as variáveis originais e os fatores, bem como a chave para o entendimento da natureza de um fator em particular" (HAIR et al., 2005. p. 90). Este mesmo autor fala que a carga deve exceder 0,70 para que o fator explique $50 \%$ da variância. 
O teste de KMO "mede o grau de correlação parcial entre as variáveis" (CORRAR; PAULO; DIAS FILHO, 2009, p. 93), sendo assim, o grau de explicação dos dados a partir das variáveis encontradas será considerado válido quando estes forem superiores a 0,50. O Teste de Bartlett's "indica se a matriz de correlação é uma matriz identidade" (CORRAR; PAULO; DIAS FILHO, 2009, p.93) devendo seu nível de significância ser próximo a 0,000.

A variância total explicada é o percentual cumulativo especificado na extração dos fatores. Hair et al. (2005) fala que em ciências sociais adota-se a variância como satisfatória quando explica $60 \%$ do total.

O Eigenvalue ou Autovalor é a "a quantia de variância explicada" (HAIR et al. 2005 , p. 90), considera-se para este critério valores acima de 1,0 . Já a Comunalidade é a quantidade de variância que uma variável compartilha.

O MSA da matriz de anti-imagem mede o "qual adequada é a aplicação da análise fatorial" (HAIR et al., 2005, p.91). Enquanto o alpha de Cronbach mede a consistência interna e a confiabilidade entre as variáveis que estão a formar o constructo, este coeficiente varia de 0 a 1 , sendo 0,70 o valor mínimo de aceitabilidade. No Quadro 2 encontra-se uma síntese com todos os critérios considerados na AFE.

Quadro 2: Síntese dos critérios da análise fatorial exploratória.

Frame 2: Summary of the criteria for exploratory factor analysis.

\begin{tabular}{|c|c|}
\hline Item & Critérios que serão considerados \\
\hline KMO & Valores maiores que 0,50 \\
\hline Sig de Bartlett's & Valores próximos a 0,000 \\
\hline Variância total & Valores acima de $60 \%$ \\
\hline Eigenvalue & Valores acima de 1,0 \\
\hline Carga fatorial & Valores acima de 0,70 \\
\hline Anti-imagem (MSA) & Valores acima de 0,50 \\
\hline Comunalidade & Valores acima de 0,50 \\
\hline Alpha de Cronbach & Valores acima de 0,70 \\
\hline
\end{tabular}

Fonte: Elaborado com base em Hair et al. (2005); Corrar; Paulo; Dias (2009); Mâroco (2010).

Source: Based on Hair et al. (2005); Run; Paulo; Dias (2009); Mâroco (2010)

Para a realização da AFE de todos os constructos o método de análise utilizado para a extração do fator foi o de componentes principais (ACP) (ESTEBAN; FERNÁNDEZ, 2001) e o método utilizado para a verificação da rotação dos fatores foi o Varimax.

Conforme os parâmetros discutidos, o constructo Valor tem os resultados da AFE apresentada na Tabela 1. Na oportunidade também são apresentados os valores descritivos de média, assimetria (valores abaixo que 3) e curtose (valores abaixo de 10) (CORRAR; PAULO; DIAS, 2009).

Inicialmente, foram inseridos na análise todos os itens que compõem este constructo. Porém, devido critérios tais como a Comunalidade abaixo do recomendado, as variáveis (valor1, valor5, valor6 e valor7) foram excluídas. Entretanto, as variáveis (valor2, valor3 e valor4) atestam a adequabilidade dos resultados da AFE para o constructo Valor, conforme os critérios definidos anteriormente. 
Tabela 1: Análise fatorial exploratória e de confiabilidade da dimensão "Valor".

Table 1: Exploratory and reliability factor analysis of the "Value" dimension.

\begin{tabular}{|c|c|c|c|c|c|c|}
\hline $\begin{array}{l}\text { Constructo e } \\
\text { variáveis } \\
\text { Valor }\end{array}$ & $\begin{array}{l}\text { Carga } \\
\text { fatorial }\end{array}$ & MSA & Comunalidade & Média & Assimetria & Curtose \\
\hline $\begin{array}{l}\text { Valor2-Eu observo } \\
\text { que ambientalmente } \\
\text { este parque é bem } \\
\text { conservado }\end{array}$ & 0,729 & $0,760^{\underline{a}}$ & 0,531 & 8,8192 & $-1,037$ & 0,381 \\
\hline $\begin{array}{l}\text { Valor3- Para mim este } \\
\text { parque possui } \\
\text { elementos atrativos }\end{array}$ & 0,844 & $0,622^{\mathrm{a}}$ & 0,712 & 8,7386 & $-1,414$ & 1,634 \\
\hline $\begin{array}{l}\text { Valor4- A experiência } \\
\text { recreativa neste } \\
\text { parque é prazerosa }\end{array}$ & 0,835 & $0,628^{a}$ & 0,697 & 9,0847 & $-1,145$ & 0,376 \\
\hline $\begin{array}{l}\text { Método de extração: An } \\
\text { Método de Rotação: Va } \\
\text { KMO (Kaiser- Meyer - } \\
\text { Bartlett's Teste of Sphe } \\
\text { Eigenvalue: } 1,940 \\
\text { Variância total: } 64,662 \% \\
\text { Alpha de Cronbach }(\alpha) \text { : } \\
\text { Fonte: Dados do estudc } \\
\text { Source: Study data, } 20\end{array}$ & $\begin{array}{l}\text { álise de comp } \\
\text { rimax } \\
\text { lkin): } 0,654 \\
\text { icity: } 116,242 \\
0,717 \\
\\
2019 .\end{array}$ & $\begin{array}{l}\text { nponentes } \\
42 \quad 5\end{array}$ & $\begin{array}{l}\text { s principais } \\
\text { Sig: } 0,000\end{array}$ & & & \\
\hline
\end{tabular}

A mesma análise foi feita para a dimensão Apoio descrita na Tabela 2.

Tabela 2: Análise fatorial exploratória e de confiabilidade da dimensão "Apoio".

Table 2: Exploratory and reliability factor analysis of the "Support" dimension.

\begin{tabular}{|c|c|c|c|c|c|c|}
\hline $\begin{array}{l}\text { Constructo e } \\
\text { variáveis } \\
\text { Apoio }\end{array}$ & $\begin{array}{l}\text { Carga } \\
\text { fatorial }\end{array}$ & MSA & Comunalidade & Média & Assimetria & Curtose \\
\hline $\begin{array}{l}\text { Apoio3- Eu sou a } \\
\text { favor do } \\
\text { desenvolvimento do } \\
\text { turismo neste } \\
\text { parque }\end{array}$ & 0,793 & $0,693^{a}$ & 0,629 & 9,1860 & $-1,779$ & 2,471 \\
\hline $\begin{array}{l}\text { Apoio4- Eu apoio um } \\
\text { maior incentivo à } \\
\text { captação de } \\
\text { visitantes para este } \\
\text { parque }\end{array}$ & 0,848 & $0,637^{a}$ & 0,719 & 9,0000 & $-1,583$ & 1,477 \\
\hline $\begin{array}{l}\text { Apoio7- Eu gostaria } \\
\text { que os turistas que } \\
\text { estão em Natal } \\
\text { visitassem este } \\
\text { parque }\end{array}$ & 0,788 & $0,699^{a}$ & 0,621 & 9,1029 & $-1,738$ & 2,013 \\
\hline
\end{tabular}

Método de extração: Análise de componentes principais

Método de Rotação: Varimax

KMO (Kaiser- Meyer - Olkin): 0,672

Bartlett's Teste of Sphericity: 118, $266 \quad$ Sig: 0,000

Eigenvalue: 1,969

Variância: $65,629 \%$

Alpha de Cronbach $(\alpha): 0,736$

Fonte: Dados do estudo, 2019.

Source: Study data, 2019. 
Aqui, da mesma forma, foram inseridas todas as variáveis, porém, ao proceder com os testes para averiguação da AFE foi apurado que apenas três variáveis apresentavam valores que justificavam a validade da análise neste constructo (apoio3, apoio4 e apoio7). Isto porque as cargas fatoriais foram superiores a 0,5, a matriz de anti-imagem (MSA) resultou em valores acima de 0,50 e as comunalidades excederam o valor de 0,50.

Na dimensão Imagem, as variáveis (Imag1, Imag2 e Imag6) foram excluídas por apresentarem resultados fora dos parâmetros da AFE, principalmente Comunalidade e Cargas fatoriais inadequadas. No entanto, na Tabela 3 podem ser observados as variáveis e seus respectivos critérios que atestam adequabilidade do constructo.

Tabela 3: Análise fatorial exploratória e de confiabilidade da dimensão "Imagem".

Table 3: Exploratory and reliability factor analysis of the "Image" dimension.

\begin{tabular}{lcccccc}
\hline $\begin{array}{c}\text { Constructo e } \\
\text { variáveis } \\
\text { Imagem }\end{array}$ & $\begin{array}{c}\text { Carga } \\
\text { fatorial }\end{array}$ & MSA & Comunalidade & Média & Assimetria & Curtose \\
\hline $\begin{array}{l}\text { Imagem3-Esse } \\
\text { parque me traz } \\
\text { boas recordações }\end{array}$ & 0,826 & $0,733^{\mathrm{a}}$ & 0,681 & 8,8531 & $-1,666$ & 1,782 \\
\hline $\begin{array}{l}\text { Imagem4- Este } \\
\text { parque me }\end{array}$ & 0,896 & $0,639^{\mathrm{a}}$ & 0,802 & 9,1556 & $-1,880$ & 2,501 \\
$\begin{array}{l}\text { lembra } \\
\text { sensações } \\
\text { agradáveis }\end{array}$ & & $0,710^{\mathrm{a}}$ & 0,703 & 9,1011 & $-1,605$ & 1,860 \\
\hline $\begin{array}{l}\text { Imagem5-Vejo } \\
\text { este parque } \\
\text { como um espaço } \\
\text { educativo }\end{array}$ & 0,839 & 0 & & & & \\
\hline
\end{tabular}

Método de extração: Análise de componentes principais

Método de Rotação: Varimax

KMO (Kaiser- Meyer - Olkin): 0,687

Bartlett's Teste of Sphericity: 190,138 Sig: 0,000

Eigenvalue: 2,187

Variância: $72,890 \%$

Alpha de Cronbach $(\alpha): 0,807$

Fonte: Dados do estudo, 2019.

Source: Study data, 2019.

Neste, os valores do KMO, da significância do teste de esfericidade de Bartlett's, do Eigenvalue e da variância total explicada garantem que os pressupostos da AFE foram atendidos, bem como, o Alpha de Cronbach, que também apresenta resultado satisfatório.

Referindo-se à Satisfação, na Tabela 4, apresentam-se os resultados dos indicadores da AFE deste constructo. 
Tabela 4: Análise fatorial exploratória e de confiabilidade da dimensão "Satisfação".

Table 4: Exploratory and reliability factor analysis of the "Satisfaction" dimension.

\begin{tabular}{lcccccc}
\hline $\begin{array}{c}\text { Constructo e } \\
\text { variáveis } \\
\text { Satisfação }\end{array}$ & $\begin{array}{l}\text { Carga } \\
\text { fatorial }\end{array}$ & MSA & Comunalidade & Média & Assimetria & Curtose \\
\hline $\begin{array}{l}\text { Sat4- O parque } \\
\begin{array}{l}\text { apresenta } \\
\text { excelente }\end{array}\end{array}$ & 0,866 & $0,703^{\underline{a}}$ & 0,749 & 7,5198 & $-0,418$ & $-0,643$ \\
$\begin{array}{l}\text { infraestrutura de } \\
\text { apoio turístico }\end{array}$ & & $0,722^{\underline{a}}$ & 0,730 & 7,9261 & $-0,351$ & $-0,800$ \\
\hline $\begin{array}{l}\text { Sat5- O parque } \\
\text { apresenta todas as } \\
\text { condições para 0 } \\
\text { lazer }\end{array}$ & 0,854 & & & & & \\
\hline $\begin{array}{l}\text { Sat6- O parque } \\
\text { possui tudo o que } \\
\text { preciso para me } \\
\text { divertir }\end{array}$ & 0,849 & $0,731^{\text {a }}$ & 0,721 & 7,4500 & $-0,674$ & $-0,165$ \\
\hline
\end{tabular}

Método de extração: Análise de componentes principais

Método de Rotação: Varimax

KMO (Kaiser- Meyer - Olkin): 0,718

Bartlett's Teste of Sphericity: $185,631 \quad$ Sig: 0,000

Eigenvalue: 2,200

Variância: $73,349 \%$

Fonte: Dados do estudo, 2019.

Source: Study data, 2019.

Todos os critérios foram atendidos, assim, as variáveis Sat4, Sat5 e Sat6 formam adequadamente o constructo Satisfação. As variáveis Sat1, Sat2, Sat 3 foram retiradas devido problemas com Comunalidade abaixo do critério, tal como, Cargas fatoriais e MSA com valores inferiores.

Por fim, na Tabela 5, expõem-se os resultados da dimensão Qualidade.

Tabela 5: Análise fatorial exploratória e de confiabilidade da dimensão "Qualidade".

Table 5: Exploratory and reliability factor analysis of the "Quality" dimension.

\begin{tabular}{lcccccc}
\hline \multicolumn{1}{c}{$\begin{array}{c}\text { Constructo } \\
\text { e variáveis }\end{array}$} & $\begin{array}{c}\text { Carga } \\
\text { fatorial }\end{array}$ & MSA & Comunalidade & Média & Assimetria & Curtose \\
\hline Qualidade & & & & & & \\
\hline $\begin{array}{l}\text { Quali1- Limpeza da } \\
\text { área }\end{array}$ & 0,750 & $0,710^{\text {a }}$ & 0,562 & 8,3889 & $-0,783$ & $-0,105$ \\
\hline Quali2- Segurança & 0,840 & $0,620^{\underline{a}}$ & 0,705 & 8,6889 & $-1,072$ & 0,353 \\
\hline Quali4- Instalações & 0,793 & $0,657^{\underline{a}}$ & 0,629 & 8,2542 & $-0,779$ & 0,127 \\
\hline
\end{tabular}

Método de extração: Análise de componentes principais

Método de Rotação: Varimax

KMO (Kaiser- Meyer - Olkin): 0,655

Bartlett's Teste of Sphericity: 101,988

Eigenvalue: 1,896

Variância: 63,217\%

Alpha de Cronbach $(\alpha)$ : 0,707

Fonte: Dados do estudo, 2019.

Source: Study data, 2019.

Como pode ser observado, as variáveis envolvidas, Quali1, Quali2 e Quali4, atendem aos pressupostos da AFE, do mesmo modo que o Alpha de Cronbach representa confiabilidade adequada. 


\section{Análise fatorial confirmatória do modelo de mensuração}

Após analisadas e verificadas a adequabilidade de todos os constructos na análise fatorial exploratória, segue-se para a análise fatorial confirmatória, esta tem como compromisso a confirmação de cada um dos constructos formados através das variáveis observáveis atribuídas na análise fatorial exploratória.

Trata-se primeiramente da avaliação da qualidade do modelo de mensuração, ou seja, a inspeção se o modelo proposto está apresentando estimativas aceitáveis. Aqui são analisados os índices de qualidade do ajustamento, isto por meio dos índices absolutos, incrementais e de parcimônia, conforme critérios definidos por Mâroco (2010) e Hair et al. (2005).

Os índices absolutos avaliam nas palavras de Hair et al. (2005, p.489) "apenas o ajuste geral do modelo", sendo neste avaliados os testes do CMIN/DF, GFI, RMSEA.

Os testes que compõem os índices incrementais são CFI, TLI, NFI, no qual avaliam "o ajuste incremental comparado com um modelo nulo" (HAIR et al., 2005, p. 496). Por fim, a medida de ajuste parcimonioso verifica a proporção do número de constructos adicionados no modelo mais o número de respondentes, um modelo muito complexo pode infringir o critério da parcimônia. Os índices mais representativos são o PGFI, PCFI e o PNFI.

Desta forma, de acordo com esses testes, seus índices de referência e os valores encontrados, confirmam-se o bom ajustamento em todas as relações, isto é, a qualidade do modelo de mensuração proposto está apropriada para fins de validação como pode ser observado na Tabela 6 .

Tabela 6: Índices de qualidade do ajustamento do modelo de mensuração.

Table 6: Quality indexes of the measurement model adjustment.

\begin{tabular}{|c|c|c|c|c|}
\hline \multicolumn{2}{|c|}{$\begin{array}{l}\text { Índices de qualidade do } \\
\text { ajustamento }\end{array}$} & $\begin{array}{l}\text { Valores do } \\
\text { modelo }\end{array}$ & $\begin{array}{c}\text { Valores de referência com } \\
\text { base em HAIR et. al. (2005); } \\
\text { MÂROCO (2010) }\end{array}$ & $\begin{array}{l}\text { Classificação do } \\
\text { índice no modelo }\end{array}$ \\
\hline \multirow{3}{*}{$\begin{array}{l}\text { Índices } \\
\text { absolutos }\end{array}$} & CMIN/DF & 1,564 & ]1;2[- ajustamento bom & Ajustamento bom \\
\hline & GFI & 0,920 & $\begin{array}{l}<0,8-\text { Ajustamento ruim } \\
{[0,8 ; 0,9[\text { - ajustamento regular }} \\
{[0,9 ; 0,95[\text { - ajustamento bom }} \\
\geq 0,95 \text { ajustamento muito bom }\end{array}$ & Ajustamento bom \\
\hline & RMSEA & 0,056 & $\begin{array}{l}>0,10 \text { - ajustamento aceitável } \\
\text { ]0,05;0,10] - ajustamento bom } \\
\leq 0,05-\text { ajustamento muito bom }\end{array}$ & Ajustamento bom \\
\hline \multirow{3}{*}{$\begin{array}{c}\text { Índices } \\
\text { incrementais }\end{array}$} & $\mathrm{CFI}$ & 0,954 & $>0,80$ - ajustamento bom & \multirow{3}{*}{ Ajustamento bom } \\
\hline & TLI & 0,938 & $>0,80$ - ajustamento bom & \\
\hline & $\mathrm{NFI}$ & 0,884 & $>0,80$ - ajustamento bom & \\
\hline \multirow{3}{*}{$\begin{array}{l}\text { Índices de } \\
\text { parcimônia }\end{array}$} & PGFI & 0,606 & \multirow{3}{*}{$\begin{array}{l}<0,6-\text { ajustamento ruim } \\
{[0,6 ; 0,8] \text { - ajustamento bom }} \\
\geq 0,8 \text { - ajustamento muito bom }\end{array}$} & \multirow{3}{*}{ Ajustamento bom } \\
\hline & PCFI & 0,717 & & \\
\hline & PNFI & 0,665 & & \\
\hline
\end{tabular}


Finalizado a verificação da qualidade do modelo de mensuração, parte-se para a validação dos constructos, neste, considera-se os indicadores de confiabilidade composta e a variância extraída (AVE). Hair et al. (2005, p.489) expressam que "a validade é o grau em que os indicadores medem com 'precisão' aquilo que eles devem efetivamente medir".

A confiabilidade composta, por sua vez, tem por finalidade apontar a consistência interna das variáveis que formam o constructo, já variância extraída (AVE) é outra medida complementar da confiabilidade, e "reflete a quantia geral de variância nos indicadores explicada pelo constructo" (HAIR et al., 2005, p.490).

Tais medidas são calculadas da seguinte forma:

(1) Confiabilidade de constructo $=\quad\left(\sum \text { cargas padronizadas }\right)^{2}$

(2) Variância extraída $=\sum$ cargas padronizadas ${ }^{2}$

$\left(\sum \text { cargas padronizadas }\right)^{2}+\sum$ erro de mensuração

$$
\sum \text { cargas padronizadas }{ }^{2}+\sum \text { erro de mensuração }
$$

O erro de mensuração dos indicadores possui a seguinte formula:

$$
\text { Erro }=1-(\text { carga padronizada })^{2}
$$

Hair et al. (2005) falam que comumente a confiabilidade do constructo deve ter como nível aceitável 0,70 , no entanto, em pesquisas em ciências sociais aceitase valores abaixo de 0,70 . Estes mesmos autores sugerem que a variância extraída deve exceder 0,50 para atender o que se propõe. Na Tabela 7 pode ser observada os resultados desses dois critérios.

Tabela 7: Estimativa dos valores de confiabilidade composta e variância extraída do modelo de mensuração.

\begin{tabular}{|c|c|c|c|c|}
\hline $\begin{array}{c}\text { Variáveis e constructo } \\
\text { (coeficientes de regressão) }\end{array}$ & $\begin{array}{c}\text { Carga } \\
\text { padronizada }\end{array}$ & Erro & $\mathbf{p}$ & $\begin{array}{c}\text { Confiabilidade composta e } \\
\text { Variância Extraída (AVE) }\end{array}$ \\
\hline Valor2↔Valor & 0,595 & - & $\star * *$ & \multirow{3}{*}{$\begin{array}{c}\text { Confiabilidade: } 0,735 \\
\text { AVE: } 0,484\end{array}$} \\
\hline Valor3 $\leftrightarrow$ Valor & 0,787 & 0,207 & $* \star \star *$ & \\
\hline Valor4 $\leftrightarrow$ Valor & 0,692 & 0,146 & $* * *$ & \\
\hline Apoio3↔Apoio & 0,638 & - & $\star * \star *$ & \multirow{3}{*}{$\begin{array}{l}\text { Confiabilidade: } 0,741 \\
\text { AVE: } 0,489\end{array}$} \\
\hline Apoio4↔Apoio & 0,770 & 0,215 & $* * *$ & \\
\hline Apoio7↔Apoio & 0,685 & 0,191 & $* \star *$ & \\
\hline Imagem3↔Imagem & 0,711 & - & $* \star \star$ & \multirow{3}{*}{$\begin{array}{l}\text { Confiabilidade: } 0,818 \\
\text { AVE: } 0,601\end{array}$} \\
\hline Imagem4↔Imagem & 0,840 & 0,112 & $* \star *$ & \\
\hline Imagem5 $\leftrightarrow$ Imagem & 0,771 & 0,096 & $* * *$ & \\
\hline Sat4↔Satisfação & 0,789 & - & *** & \multirow{3}{*}{$\begin{array}{l}\text { Confiabilidade: } 0,817 \\
\text { AVE: } 0,599\end{array}$} \\
\hline Sat5↔Satisfação & 0,747 & 0,086 & $* \star \star *$ & \\
\hline Sat6 $\leftrightarrow$ Satisfação & 0,786 & 0,104 & $* \star \star$ & \\
\hline Quali1 $\leftrightarrow$ Qualidade & 0,425 & - & $* * *$ & \multirow{3}{*}{$\begin{array}{l}\text { Confiabilidade: } 0,676 \\
\text { AVE: } 0,430\end{array}$} \\
\hline Quali2 $\leftrightarrow$ Qualidade & 0,599 & 0,254 & $* * *$ & \\
\hline Quali4 $\leftrightarrow$ Qualidade & 0,867 & 0,422 & $* \star \star$ & \\
\hline
\end{tabular}

Table 7: Estimated values of composite reliability and variance extracted from the measurement model.

***Relação altamente significante

Fonte: Dados do estudo, 2019.

Source: Study data, 2019. 
Em termos de confiabilidade, apenas o constructo Qualidade desvia um pouco do valor recomendado $(0,676)$, porém, ao considerar que o estudo se trata de uma pesquisa em ciências sociais, aceita-se este valor. $E$ em termos de variância extraída, os constructos Valor $(0,484)$ e Apoio $(0,489)$ estão no limite do critério sugerido, e o constructo Qualidade tem o valor um pouco menor $(0,430)$. Apesar desses valores levemente abaixo da orientação, ainda assim, os indicadores podem ser considerados suficientes na especificação do modelo de mensuração proposto, pois não violam gravemente a sugestão dos autores.

Feito todas as etapas anteriormente descritas, o modelo de mensuração encontra-se validado para fins desse estudo.

\section{Considerações finais}

Este trabalho tratou da validação empírica de um instrumento de mensuração dos fatores antecedentes do apoio ao desenvolvimento turístico em uma UC. Esta validação representa as relações entre as variáveis observáveis e os fatores propostos, que quanto a isso, foram verificados através de estatística multivariada, análise fatorial exploratória e análise fatorial confirmatória.

$\mathrm{Na}$ análise fatorial exploratória foram aplicados critérios para a verificação da adequabilidade das variáveis que formavam a dimensão, e na confirmatória avaliouse a qualidade do ajustamento do modelo de mensuração, tal como a confiabilidade e a variância extraída das variáveis. Feito todos os testes e examinando que os indicadores apresentavam parâmetros suficientes para a especificação do modelo, atestou-se a validação. Com isso, há possibilidade de utilização deste instrumento em pesquisas com residentes que busquem compreender o Valor, a Imagem, a Satisfação, a Qualidade e o Apoio ao desenvolvimento do turismo atribuído a UC, mais especificamente a Parques Naturais.

Feito isso, em análise posterior tais resultados serão utilizados para aplicação e utilização em Modelagem de Equações Estruturais com a finalidade de compreender as inter-relações entre os constructos e a influência deles sobre o apoio ao desenvolvimento do turismo.

De maneira interpretativa, o conjunto de variáveis relacionadas pelos residentes atribuído ao constructo Valor, trata-se da observação da conservação ambiental, da existência de elementos atrativos e por ser prazerosa a experiência recreativa no parque. O que de maneira teórica vai de encontro com as prerrogativas de UC (BRASIL, 2000) e com as ideias de Passold e Kinker (2010) sobre a utilidade, a relevância e a importância de parques naturais através da conservação, da atratividade e da possibilidade do usufruto do lazer.

Já o constructo Imagem, é formado pelas boas recordações que o local proporciona, tal como, às sensações agradáveis recordadas pelos visitantes e por ser possível enxergá-lo como um local educativo. Características próprias de UC (IUCN, 2011) e que reforçam o discurso de Ramkissoon e Nunkoo (2011) sobre as imagens positivas serem acompanhadas de um maior nível de apoio ao desenvolvimento do turismo.

Enquanto as variáveis relacionadas à Satisfação, refere-se ao fato do parque apresentar excelente infraestrutura de apoio ao turismo, condições ao lazer e 
possibilidade de diversão. Atributos que devem estar relacionados a todos os Parques Naturais como afirma o SNUC (BRASIL, 2000).

Á medida que, o constructo Qualidade é formado através da limpeza da área, da segurança e das instalações, condições fundamentais para áreas de uso público, e possibilitando, conforme afirmam Bushell et al. (2007), que a alta qualidade na experiência proporcione o aumento do interesse na proteção e conservação de áreas protegidas.

E por fim, as variáveis que foram relacionadas pelos residentes ao constructo Apoio ao desenvolvimento do turismo, estão atreladas à concordância do crescimento da atividade no parque, do incentivo à captação de visitantes e do desejo da vinda de turistas. O que reforça o discurso de Ramkissoon e Nunkoo (2011) e Garcia, Vázquez e Macías (2015) ao afirmarem que os residentes são mais propensos a apoiar o turismo desde que percebam os benefícios.

Torna-se importante ressaltar a relevância de estudos desta natureza, visando investigar variáveis observáveis na formação de constructos, verificar se estão medindo o que devem medir, validá-los e atestar a sua correlação, tendo em vista o desenvolvimento e aperfeiçoamento de instrumentos de pesquisa adequados e coerentes.

Apresenta-se como limitações da pesquisa o fato da coleta não ter sido feita em um tempo maior, contemplando a estação de férias, momento de maior fluxo de pessoas no parque, embora o número de respondentes neste trabalho atenda ao recomendado na literatura. Sugere-se assim que as próximas pesquisas com essa perspectiva abranjam um tempo maior de coleta de dados.

\section{Referências}

ALAM, Md. S.; PARAMATI, S. R. The impact of tourism on income inequality in developing economies: Does Kuznets curve hypothesis exist? Annals of Tourism Research, v.61, p.111-126, 2016.

ANDERECK, K.; VALENTINE, K.; KNOPF, R.; VOGT, C. Residents' perceptions of community tourism impacts. Annals of Tourism Research, v.32, n.4, pp.1056-1076, 2005.

AJZEN, I.; FISHBEIN, M. Attitude-behavior relations: A theoretical analysis and review of empirical research. Psychological Bulletin, v.84, n.5, pp-888-918, 1977.

AP, J. Residents' perceptions on Tourism impacts. Annals of Tourism Research,19 v.4, pp-665-690, 1992.

AP., J.; CROMPTON, J. Developing and testing a tourism impact scale. Journal of Travel Research, v.37, pp-120-130, 1998.

BALLANTYNE, R.; PACKER, J.; HUGHES, K. Environmental awareness, interests and motives of botanic gardens visitors: Implications for interpretive practice. Tourism Management, v.29, pp-439-444, 2008.

BEERLI, A.; MARTíN, J. Factors influencing destination image. Annals of Tourism Research, v.31, n.3, pp.657-681, 2004.

BESTARD, A. B.; NADAL, J. R. Modelling environmental attitudes towards tourism. Tourism Management, v.28, n.3, pp-688-695, 2007. 
BOWERS, J. Developing sustainable tourism through ecomuseology: a case study in the Rupununi region of Guyana. Journal of Sustainable Tourism, v.24, pp-758782, 2016.

BLANCO-CERRADELO, L.; GUEIMONDE-CANTO, A.; FRAIZ-BREA, J.A.; DIEGUEZ-CASTRILLON, M. I. Dimensions of destination competitiveness: analyses of protected areas in Spain. Journal of Cleaner Production, v.177, pp-782-794., 2018.

BUSHELL, R.; STAIFF, R; EAGLES, P.F.J. Tourism and protected areas: benefits beyond boundaries. In: BUSHELL, R., EAGLES, P.F.J. (org.). Tourism and protected areas: benefits beyond boundaries. Wallingford: CABI Publishing, 2007.

BRASIL. Ministério do Meio Ambiente. Sistema Nacional de Unidades de Conservação- SNUC. 2000. Disponível em: <http://www.mma.gov.br/images/ar quivos/areas protegidas/snuc/Livro\%20SNUC\%20PNAP.pdf>. Acesso em: 20/07/18.

BRASIL. Ministério do Meio Ambiente. Visitação em parques nacionais sobe 238\%. 2016. Disponível em: <http://www.mma.gov.br/index.php/comunicacao lagencia-informma?view=blog\&id=1427> . Acesso em 02 de junho de 2017.

BRASIL. Embratur. 2018. Disponível em: <http://www.embratur.gov.br/piembraturnew/opencms/salalmprensa/noticias/arquivos/Site de noticias da Argentina indica visita a parques naturais brasileiros.html>. Acesso em 28 de agosto de 2018.

BRASIL. Ministério do Turismo. Anuário Estatístico de turismo 2018- Ano base 2017. MTUR, Brasília, 2018.

BRILHA, J. Patrimônio geológico e geoconservação: a conservação da natureza na sua vertente geológica. Viseu: Palimage Editores, 2005.

CHEN, C.-F.; TSAI, D. How destination image and evaluative factors affect behavioral intentions?. Tourism Management, v.28, n.), pp-1115-1122, 2007.

CHEN, C.-F.; CHEN, F.-S. Experience quality, perceived value, satisfaction and behavioral intentions for heritage tourists. Tourism Management, v.31, n.1, pp-2935, 2010.

CORRAR, L.; PAULO, E; DIAS FILHO, J. M. Análise Multivariada: para os cursos de administração, ciências contábeis e economia. São Paulo: Atlas, 2009.

DIEGUES, A. C. O Mito Moderno da Natureza Intocada. São Paulo: Hucitec. 1998.

DOUROJEANNI, M. Associação o Eco. 2015. Disponível em: $<$ http://www.oeco.org.br/colunas/marc-dourojeanni/28858-sobre-a-origem-do-termounidade-de-conservacao $>$. Acesso em 10/05/17.

EAGLES, P.; MCCOLL, S. Tourism in national parks and protected areas: planning and management. New York: Cabi Publishing, 2002.

ESTEBAN, I. G.; FERNÁNDEZ, E. A. Fundamentos y técnicas de investigacion comercial. Madrid: ESIC Editora, 2001.

FORNELL, C.; JOHNSON, M.; ANDERSON, E.; CHA, J.; BRYANT, B. The american customer satisfaction Index: nature, purpose, and findings. Journal of Marketing, v.60, n.4, pp-7-18, 1996.

GARCIA, F. A.; VÁZQUEZ, A.; MACÍAS, R. Resident's attitudes toward the impacts of Tourism. Tourism Management Perspective, v.13, pp-33-40, 2015. 
GURSOY, D.; RUTHERFORD, D. Host attitudes toward tourism: An improved structural model. Annals of Tourism Research, v.31, n.3, pp-495-516, 2004.

GURSOY, D.; JUROWSKI, C.; UYSAL, M. Resident attitudes: A structural modeling approach. Annals of Tourism Research, v.29, n.1, pp-79-105, 2002.

GURSOY, D.; BOGAN, E.; DEDEOGLU, B. B.; ÇALISKAN, C. Residents' perceptions of hotels' corporate social responsibility initiatives and its impact on residents' sentiments to community and support for additional tourism development. Journal of Hospitality and Tourism Management, v.39, pp-117-128, 2019.

GRAY, M. Geodiversity: valuing and conserving abiotic nature. Chichester: John Wiley and sons, 2004

HAIR, J.; ANDERSON, R.; TATHAM, R.; BLACK, W. Análise Multivariada de dados. Porto Alegre: Bookman, 2005.

HALEY, A.; SNAITH, T.; MILLER, G. The social impacts of tourism: A case study of Bath, UK. Annals of Tourism Research, v.32, n.3, pp-647- 668, 2005.

IUCN._Las Areas Protegidas de América Latina: Situacion actual y perspectiva para el futuro._IUCN, 2011.

JO, W.; LEE, C.; REISINGER, Y. Behavioral intentions of international visitors to the Korean hanok guest houses: Quality, Value and Satisfacion. Annals of Tourism Research, v.41, pp-89-109, 2014.

KASHYAP, R.; BOJANIC, D. A structural analysis of value, quality and price perception of business and leisure travellers. Journal of Travel Research, v.39, n.1, pp-45-51, 2000.

LEE, C.; LEE, Y.; LEE, B. Korea's destination image formed by the 2002 word cup. Annals of Tourism Research, v.32, n.4, pp-839-858, 2005.

LEE, T. H. Influences analysis of community residente support for sustainable tourism development. Tourism Management, v.34, pp-37-46, 2013.

LEE, C.- K.; BACK, K.- J. Examining structural relationships among perceived impact, benefit, and support for casino development based on 4 year longitudinal data. Tourism Management, v.27, pp-466-480, 2006.

LEPP, A. Residents' attitudes towards tourism in Bigodi Village, Uganda. Tourism Management, v.28, n.3, pp-876-885, 2007.

LINDBERG, K.; JOHNSON, R. Modeling resident attitudes toward Tourism. Annals of Tourism Research, v.24, n2, pp-402-424, 1997.

LIU, J.; VAR, T. Resident attitudes toward tourism impacts in Hawaii. Annals of Tourism Research, v.13, n.2, pp-193-214, 1986.

LIU, J.; SHELDON, P.; VAR, T. Resident perception of the environmental impacts of Tourism. Annals of Tourism Research, v.14, n.1, pp-17-37, 1987.

MARÔCO. J. Análise de Equações Estruturais: Fundamentos teóricos, software e aplicações. Perô Pinheiro: Report Nunber, 2010.

MATHEUS, F. S; RAIMUNDO, S. Os resultados das políticas públicas de ecoturismo em Unidades de Conservação no Brasil e no Canadá. Revista Brasileira de Pesquisa em Turismo, v.11, n.3, pp-454-479, 2017. 
MOGHAVVEMI, S.; WOOSNAM, K.; PARAMANATHAN, T.; MUSA, G.; HAMZAH, A. The effect of residents' personality, emotional solidarity, and community commitment on support for tourism development. Tourism Management, v.63, pp-242-254, 2017.

MOREIRA. J. Pesquisa de uso e recreação em Unidade de Conservação. Universidade Estadual de Ponta Grossa, 2016.

MCCORNIK, J. Rumo ao Paraíso: a história do movimento ambientalista. 1992. Disponível em:<https://pt.scribd.com/doc/55372947/McCORMICK-John-Rumo-aoParaiso-A-historia-dos-movimentos-ambientalistas >. Acesso: 17/05/17.

MURPHY, P. Community attitudes to tourism: A comparative analysis. Tourism Management, v.2, n.3, pp-189-195, 1981.

MURPHY, P.; PRITCHARD, M. P.; SMITH, B. The destination product and its impact on traveler perceptions. Tourism Management, v.21, n.1, pp-43-52, 2000.

NASCIMENTO, M.; RUCHKYS, U.; MANTESSO-NETO, V. Geodiversidade, Geoconservação e Geoturismo: trinômio importante para a proteção do patrimônio geológico. São Paulo: SBGeo, 2008.

NUNKOO, R.; RAMKISSOON, H. Developing a community support model for tourism. Annals of Tourism Research, v.38, n.3, pp-964-988, 2011.

NUNKOO, R.; RAMKISSOON, H. Power, trust social Exchange and community support. Annals of Tourism Research, v.39, n.2, pp-997-1023, 2012.

NUNKOO, R.; GURSOY, D. Residents'support for tourism: An identity perspective. Annals of tourism research, v.39, n.1, pp-243-268, 2012.

$\mathrm{OH}, \mathrm{H}$. Service quality, customer satisfaction, and costumer value: A holistic perspective. International Journal of hospitality Management, v.18, n.1, pp-67-82, 1999.

$\mathrm{OH}, \mathrm{H}$. Diners' perceptions of quality, value and satisfaction. Cornell Hotel and Restaurant Administration Quarterly, v.41, n.3, pp-58-66, 2000.

OVIEDO-GARCíA, M. Á.; VEGA-VÁZQUEZ, M.; CASTELLANOS-VERDUGO, M.; ORGAZ-AGUERA, F. Tourism in protected areas and the impact of servicescape on tourist satisfaction, key in sustainability. Journal of Destination Marketing \& Management, v.12, pp-74-83, 2019.

PASSOLD, A. J.; KINKER, S.M. S. Visitação em unidades de conservação. In PHILIPPI JR. A.; RUSCHMANN, D. V. M. Gestão ambiental e sustentabilidade no turismo. São Paulo: Manole, 2010.

PETRICK, J. The roles of quality, perceived value and satisfaction in predicting cruise passengers' behavioral intentions. Journal of Travel Research, v.42, pp-397407, 2004.

PERDUE, R.; LONG, P.; ALLEN, L. Rural resident tourism perceptions and attitudes. Annals of Tourism Research, v. 14, n.3, pp-420-429, 1987.

RAMKISSOON, H., NUNKOO, R. City image and perceived tourism impact: evidence from Port Louis, Mauritius. International Journal of Hospitality, v.12, n.2, pp-123143, 2011. 
ROCHA, L. G. M. Os Parques Nacionais do Brasil e a Questão Fundiária: o caso do Parque Nacional da Serra dos Órgãos. Dissertação de Mestrado. Programa de Pós Graduação em Ciência Ambiental da Universidade Federal Fluminense. Rio de Janeiro, 2002.

STYLIDIS, D.; BIRAN, A.; SIT, J.; SZIVAS, E. Residents'support for tourism development: The role of residents' place image and perceived tourism impacts. Tourism Management, v.45, pp-260-274, 2014.

STYLIDIS, D.; SHANI, A.; BELHASSEN, Y. Testing na integrated destination image model across residents and tourists. Tourism Management, v.58, pp-184-195, 2017.

SHIRAISHI, J. C. Conflitos ambientais em Unidades de Conservação: Percepções sobre a Reserva Biológica da Contagem- DF. Dissertação de Mestrado. Universidade de Brasília, 2011.

SCHROEDER, T. The relationship of residents' image of their state as a tourism destination and their support for tourism. Journal of Travel Research, v.34, pp-7173, 1996.

SCHWEINSBERG, S.; DARCY, S.; CHENG, M. The agenda setting power of news media in framing the future role of tourism in protected areas. Tourism Management, v.62, pp-241-252, 2017.

STRICKLAND-MUNRO, J.; ALISSON, H.; MOORE, S. Using resilience concepts to investigate the impacts of protected área tourism on communities. Annals of Tourism Research, v.37, n.2, pp-499-519, 2010.

VALLEJO, L. R. Uso público em áreas protegidas: atores, impactos, diretrizes de planejamento e gestão. Revista Eletrônica Uso público em Unidades de Conservação, v.1, n;1, pp-13-26, 2013.

VALLEJO, L. R. Unidades de Conservação : Uma discussão teórica á luz dos conceitos de território e de politicas públicas. Revista Geografia, Universidade Federal Fluminense, 2002.

VARGAS-SÁNCHEZ, A.; PORRAS-BUENO, N.; PLAZA-MEJÍA, M. Explaining residents' attitudes to tourism: Is a universal model possible? Annals os Tourism Research, v.38, n.2, pp-460-480, 2011.

ZEITHAML, V. Consumer perceptions of price, quality, and value: A means-end model and synthesis of evidence. Journal of Marketing, v.52, n.3, pp-2-22, 1988.

WANG, X.; ZHANG, J.; GU, C.; ZHEN, F. Examining antecedentes and consequences of tourist satisfaction: A structural modeling approach. Tsinghua Science and Technology, v. 14, n.3, pp-397-406, 2009.

WOODRUFF, R. Customer Value: The next source for competitive advantage. Academy of marketing Science, v.27, n.2, pp-139-153, 1997.

WORLD ECONOMIC FORUM. The travel e tourism competitiveness report 2017: paving the way for a more sustainable and inclusive future. Geneva: Word Economic Forum, 2017.

ZINDA, J. A. Tourism dynamos: Selective commodification and developmental conservation in China's protected areas. Geoforum, v.78, pp-141-152, 2017. 


\section{Agradecimentos}

Trabalho originado de dissertação de mestrado do Programa de Pós Graduação em Turismo da Universidade Federal do Rio Grande do Norte e contou com o apoio financeiro através de bolsa de pesquisa da CAPES.

Carla Stefânia Cabral de Medeiros Santana: Universidade Federal do Rio Grande do Norte, Natal, RN, Brasil.

Email: carlastefaniasantana@yahoo.com.br

Link para o currículo Lattes: http://lattes.cnpq.br/0018480906444662

Marcos Antônio Leite do Nascimento: Universidade Federal do Rio Grande do Norte, Natal, RN, Brasil.

Email: marcos@geologia.ufrn.br

Link para o currículo Lattes: http://lattes.cnpq.br/5356037408083015

Sérgio Marques Junior: Universidade Federal do Rio Grande do Norte, Natal, RN, Brasil.

Email: sergio@ct.ufrn.br

Link para o currículo Lattes: http://lattes.cnpq.br/3095760811566254

Data de submissão: 13 de setembro de 2019

Data de recebimento de correções: 06 de fevereiro de 2020

Data do aceite: 06 de fevereiro de 2020

Avaliado anonimamente 